\title{
Magnetospheric plasma boundaries: a test of the frozen-in magnetic field theorem
}

\author{
R. Lundin ${ }^{1}$, M. Yamauchi ${ }^{1}$, J.-A. Sauvaud ${ }^{2}$, and A. Balogh ${ }^{3}$ \\ ${ }^{1}$ Swedish Institute of Space Physics, Kiruna, Sweden \\ ${ }^{2}$ CESR-CNRS, Toulouse, France \\ ${ }^{3}$ Imperial College, London, UK
}

Received: 19 January 2005 - Revised: 1 June 2005 - Accepted: 20 June 2005 - Published: 14 October 2005

\begin{abstract}
The notion of frozen-in magnetic field originates from H. Alfvén, the result of a work on electromagnetichydrodynamic waves published in 1942. After that, the notion of frozen-in magnetic field, or ideal MHD, has become widely used in space plasma physics. The controversy on the applicability of ideal MHD started in the late 1950s and has continued ever since. The applicability of ideal MHD is particularly interesting in regions where solar wind plasma may cross the magnetopause and access the magnetosphere. It is generally assumed that a macroscopic system can be described by ideal MHD provided that the violations of ideal MHD are sufficiently small-sized near magnetic x-points (magnetic reconnection). On the other hand, localized departure from ideal MHD also enables other processes to take place, such that plasma may cross the separatrix and access neighbouring magnetic flux tubes. It is therefore important to be able to quantify from direct measurements ideal MHD, a task that has turned out to be a major challenge.

An obvious test is to compare the perpendicular electric field with the plasma drift, i.e. to test if $\boldsymbol{E}=-\boldsymbol{v} \times \boldsymbol{B}$. Yet another aspect is to rule out the existence of parallel (to B) electric fields. These two tests have been subject to extensive research for decades. However, the ultimate test of the "frozen-in" condition, based on measurement data, is yet to be identified. We combine Cluster CIS-data and FGM-data, estimating the change in magnetic flux $(\delta \boldsymbol{B} / \delta t)$ and the curl of plasma $-\boldsymbol{v} \times \boldsymbol{B}(\nabla \times(\boldsymbol{v} \times \boldsymbol{B}))$, the terms in the "frozen-in equation". Our test suggests that ideal MHD applies in a macroscopic sense in major parts of the outer magnetosphere, for instance, in the external cusp and in the high-latitude magnetosheath. However, we also find significant departures from ideal MHD, as expected on smaller scales, but also on larger scales, near the cusp and in the magnetosphere-boundary layer. We discuss the importance of these findings.
\end{abstract}

Keywords. Magnetospheric physics (Magnetopause, cusp and boundary layers; Solar wind-magnetosphere interactions) - Space plasma physics

Correspondence to: R. Lundin

(rickard.lundin@irf.se)

\section{Introduction}

H. Alfvén noted, in connection with his discovery of magnetohydrodynamic (MHD) waves (Alfvén, 1940), that the motion of matter may couple to the deformation of the magnetic field such that the field lines follow the motion of matter. Alfvén denoted this "frozen-in magnetic field lines". The "frozen-in magnetic field lines" theorem and its corollary "ideal MHD" are valid in plasmas under special conditions. The theorem, or law, may be derived from Ohm's law and Maxwells equations. Assuming infinite conductivity $(\sigma)$ along magnetic field lines implies a high magnetic Reynold number $\left(R_{m}=\mu_{0} \sigma l_{c} v_{c} \gg 0\right)$ and $\delta \boldsymbol{B} / \delta t=\nabla \times(\boldsymbol{v} \times \boldsymbol{B})$. This leads to the following relation for Farady's law in integral form:

$$
\frac{d \Phi}{d t}=\iint\left(\frac{\partial \boldsymbol{B}}{\partial t}-\nabla \times(\boldsymbol{v} \times \boldsymbol{B}) d \boldsymbol{S},\right.
$$

where $\Phi$ is the magnetic flux through a variable surface, its contours at each point moving with speed $v$, and $B$ is the magnetic field. $d \Phi / d t \approx 0$ in Eq. (1) now implies that the magnetic flux $\Phi$ through every surface remains constant. The magnetic field lines through the surfaces will then also be the same along the entire flux tube. The perpendicular motion of the plasma is everywhere following the magnetic field line, i.e.

$-(\boldsymbol{v} \times \boldsymbol{B})=\boldsymbol{E}$,

where $E$ is the electric field. Notice that a nonzero $d \Phi / d t$ implies electromotive forcing by $E_{e m f}$, i.e. from the FaradayHenry law:

$E_{\text {emf }}=-\Phi \frac{d \Phi}{d t}$.

A negative $d \Phi / d t$ implies induction, i.e. $\nabla \times(\boldsymbol{v} \times \boldsymbol{B}>\partial \boldsymbol{B} / \partial t$ and plasma forcing by, for example, electric currents inducing magnetic fields (Ampéres law) that oppose ambient magnetic flux variations. Conversely, a positive $d \Phi / d t$ implies the reverse, i.e. magnetic field changes produce currents that may lead to plasma acceleration. Therefore, $d \Phi / d t$ defines the cause-effect relationship between perturbation magnetic fields and currents in a plasma. 
"Frozen-in magnetic field lines", or "ideal MHD", has been quite successful in modelling large-scale plasma phenomena in the Earth's magnetosphere and on the Sun, the advantage being that plasma motion may be described by moving magnetic field lines. H. Alfvén, the founder of MHD, raised great concern about ideal MHD, or rather what he considered the misuse of MHD (e.g. Alfvén, 1958, 1976, 1981). One of the most important deviations from ideal MHD is magnetic reconnection, which is the merging of magnetic field lines, as invented by Sweet (1958) and Parker (1957a), and later applied to the Earth's magnetosphere by Dungey (1961); it has become a central paradigm in space plasma physics. The conversion of magnetic energy to plasma kinetic energy, published by Paschmann et al. (1979) and Sonnerup et al. (1981), may be considered the first indirect proof for magnetic reconnection, which requires violation of ideal MHD at a finite volume in space (Schindler, 1988), where magnetic field lines can merge (Vasyliunas, 1975). However, the physics involved in the magnetic field line merging remains poorly understood. Violation of ideal MHD has a broader context, though, allowing for a multitude of physical processes related to energy and momentum transfer. It should, first of all, be a question about the plasma physical processes promoting violation of ideal MHD, rather than their macroscopic influences. Moreover, what are the principal observable parameters that characterize such a breakdown? Recent observations of electric and magnetic fields in the diffusion region (e.g. Mozer et al., 2002) are consistent with the action of the Hall effect in collisionless reconnection. However, more high-resolution observations, combining magnetic and electric field data with particle data, are certainly required to elucidate the physics in the diffusion region.

Besides the breakdown of the single fluid concept of MHD (Yamauchi and Blomberg, 1997), departure from ideal MHD can be identified in two ways. First, by the existence of parallel (with B) electric fields $\left(E_{\|}\right)$inherently requiring a finite parallel conductivity along $B$. The debate on this issue dates back to the 1950s (Alfvén, 1958). The existence of $E_{\|}$along auroral magnetic field lines, upward directed, as well as downward directed, is recognized today (see, e.g. Paschmann et al., 2003 for a review). Since $E_{\|}$occurs on auroral field lines up to altitudes of several Earth radii, there are good reasons to believe that $E_{||}$also exists at other places in the Earth's magnetosphere.

Secondly, departure from ideal MHD originates from the fact that $E$ in Eq. (2) refers to the motional electromotive force (emf), i.e. the perpendicular plasma motion across B. However, the perpendicular drift is due to more than just the electric drift. Perpendicular drift is also governed by plasma inertia, pressure gradients and anisotropies, as implied from the first order drift theory (e.g. Alfvén, 1950; Parker, 1957b; Alfvén and Fälthammar, 1963),

$\boldsymbol{v}_{\perp}=\frac{\boldsymbol{B}}{B^{2}} \times\left[\left(\boldsymbol{E}+\frac{m}{q} \frac{d \boldsymbol{v}_{0}}{d t}\right)+\frac{\nabla P_{\perp}}{q n}+\frac{\left(P_{\|}-P_{\mid}\right)}{q n}(\boldsymbol{b} \cdot \nabla) \boldsymbol{b}\right]$.
Observations of species-dependent differential ion drift, induced by, for example, pressure gradients and ion inertia, leading to drift velocities comparable to the electric drift, has been presented (e.g. Lundin et al., 1987, 2003). This implies that the motional emf and the electric field are not identical, and ideal MHD breaks down for ions. Even if the electric drift dominates for electrons, charging may result from the difference in ion and electron drift, and electric currents are produced.

Regions where ideal MHD breaks down are important because they constitute regions of energy and momentum transfer, for example, transfer of solar wind/magnetosheath energy into the magnetosphere - where electromagnetic energy is converted to plasma kinetic energy, and vice versa. In MHD terminology the conversion of energy follows from (e.g. Cole, 1961; Chen, 1984):

$\boldsymbol{J}^{\prime} \cdot \boldsymbol{E}^{\prime}=\boldsymbol{J} \cdot(\boldsymbol{E}+(\boldsymbol{v} \times \boldsymbol{B})) \neq 0$,

where $\boldsymbol{J}^{\prime}$ and $\boldsymbol{E}^{\prime}$ mark the polarization current and polarization electric field in the frame of reference of moving plasma, $\boldsymbol{J} \cdot \boldsymbol{E}<0$ marks a dynamo and $\boldsymbol{J}^{\prime} \cdot \boldsymbol{E}^{\prime}>0$ marks a load. Since $\boldsymbol{J}^{\prime}=\boldsymbol{J}$, i.e. the current is independent of the frame of reference we have:

$\boldsymbol{E}^{\prime}=\boldsymbol{E}+(\boldsymbol{v} \times \boldsymbol{B}) \neq 0$.

The simple frozen-in picture, that is $\boldsymbol{E}^{\prime}=0$, automatically loses validity by this type of energy-conversion argument in the snapshot of a dynamic situation.

The question is, where does ideal MHD apply and where doesn't it? Can one find an observable plasma marker for ideal MHD? From Eq. (1) we find that the frozen-in theorem requires that spatial variations of plasma, i.e. $\nabla \times(\boldsymbol{v} \times \boldsymbol{B})$, must match temporal variations of $\boldsymbol{B}$ within a surface area $S$. In a fixed frame of reference (e.g. GSE) the $\nabla \times(\boldsymbol{v} \times \boldsymbol{B})$ vector should correlate with $B$ for ideal MHD.

A proper test of Eq. (1) includes determination of $\delta \boldsymbol{B} / \delta t$ and $\nabla \times(\boldsymbol{v} \times \boldsymbol{B})$. The $\nabla \times(\boldsymbol{v} \times \boldsymbol{B})$ represents a spatially averaged quantity within a flux tube of dimension at least two ion Larmor radii, while the local magnetic field $\mathrm{B}$ is an inherently integral quantity. The $\nabla \times(\boldsymbol{v} \times \boldsymbol{B})$ is therefore the critical term in the test.

Testing ideal MHD by Eq. (1) may be compared with another test criteria, the plasma drift Eq. (3) based on the $-\boldsymbol{v} \times \boldsymbol{B}$ for two energy regimes (see, e.g. Lundin et al., 2003). In the next section we use Cluster CIS and FGM data to test the above-mentioned two methods.

\section{Observational test of ideal MHD}

Observational tests of ideal MHD can be made by different means: first, as described below, by independently measured magnetic field and plasma drift velocity (Eq. 1), and second, by direct comparison of $\boldsymbol{E}$ and $\boldsymbol{v} \times \boldsymbol{B}$. However, the Electron Drift Instrument (EDI) does not provide data for low and variable magnetic fields and EFW only measures the two components of the electric field in the spin plane. The third 


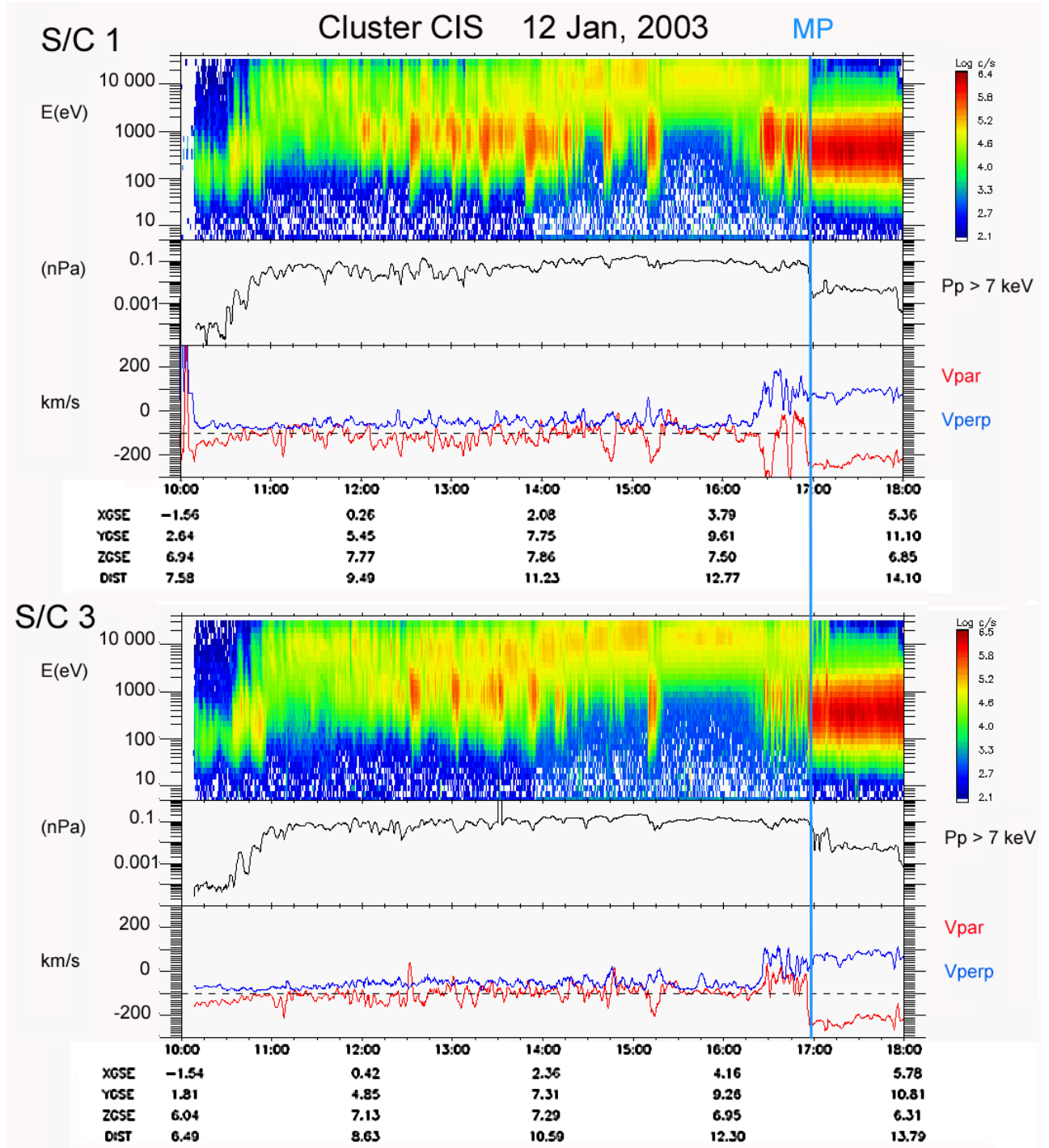

Fig. 1. Overview plot of Cluster s/c 1 and s/c 3 CIS-data on 12 January 2003. The panels illustrate, from top to bottom: energy-time ion spectrograms, ion pressure for energies greater than $10 \mathrm{keV}$, and parallel (red) and perpendicular (blue) ion flow velocities, respectively. Blue vertical lines illustrate the almost simultaneous encounter of the magnetopause boundary for s/c 1 and s/c 3 .

test, to verify the existence of field-aligned electric fields (i.e. $\boldsymbol{E} \cdot \boldsymbol{B}=0$ ) is not feasible here. Considering this it appears simpler, at least in a first round, to compute the ion drift velocities for different energy ranges and to compare the resulting $\boldsymbol{v} \times \boldsymbol{B}$. For ideal MHD, these $\boldsymbol{v} \times \boldsymbol{B}$ quantities should be equal.

The second test is to use Eq. (1) and determine the time derivative of $B$ and the $\boldsymbol{E}_{\text {emf }}=-\boldsymbol{v} \times \boldsymbol{B}$ along the spacecraft orbit. A proper test of "ideal MHD" requires integration over a surface area $S$ - a task which is difficult, if not impossible, for Cluster, because of measurement limitations. Instead, we use what may be termed as the "frozen-in equation" that holds for high Magnetic Reynolds numbers $\left(\mathrm{R}_{m} \gg 1\right)$ :

$\frac{\partial \boldsymbol{B}}{\partial t}=\nabla \times(\boldsymbol{v} \times \boldsymbol{B})$.

Taking $\delta \boldsymbol{B} / \delta t$ from the magnetic field data and $\nabla \times \boldsymbol{E}_{\text {emf }}$ from the ion data we obtain a test of the frozen-in equation. $\delta \boldsymbol{B} / \delta t$ is obtained by subtracting a background $B$-field component assumed to be quasi-steady, i.e. varying very slowly along the s/c orbit. We assume that the offset $B$-component along the spacecraft orbit is everywhere due to temporal variations of $B$. Quasi-steady spatial variations of $B$ along the $\mathrm{s} / \mathrm{c}$ orbit are assumed to be embedded in the background $B$-component. An ambiguity between spatial and temporal variations from single point measurement is inevitable because $d / d t=\delta / \delta t+(\boldsymbol{u} \cdot \nabla)$, i.e. moving spatial structures will appear as "temporal" in the s/c frame of reference. On the other hand, inherent in this analysis is that spatially moving structures would also comply with the "frozen-in equation" (if it holds). We will return to this problem in discussing the small-scale variations. As for large-scale variations we have selected cases where the magnetopause and boundary layer are relatively stable in time and space, at least based on data from s/c 1, 3 and 4. 


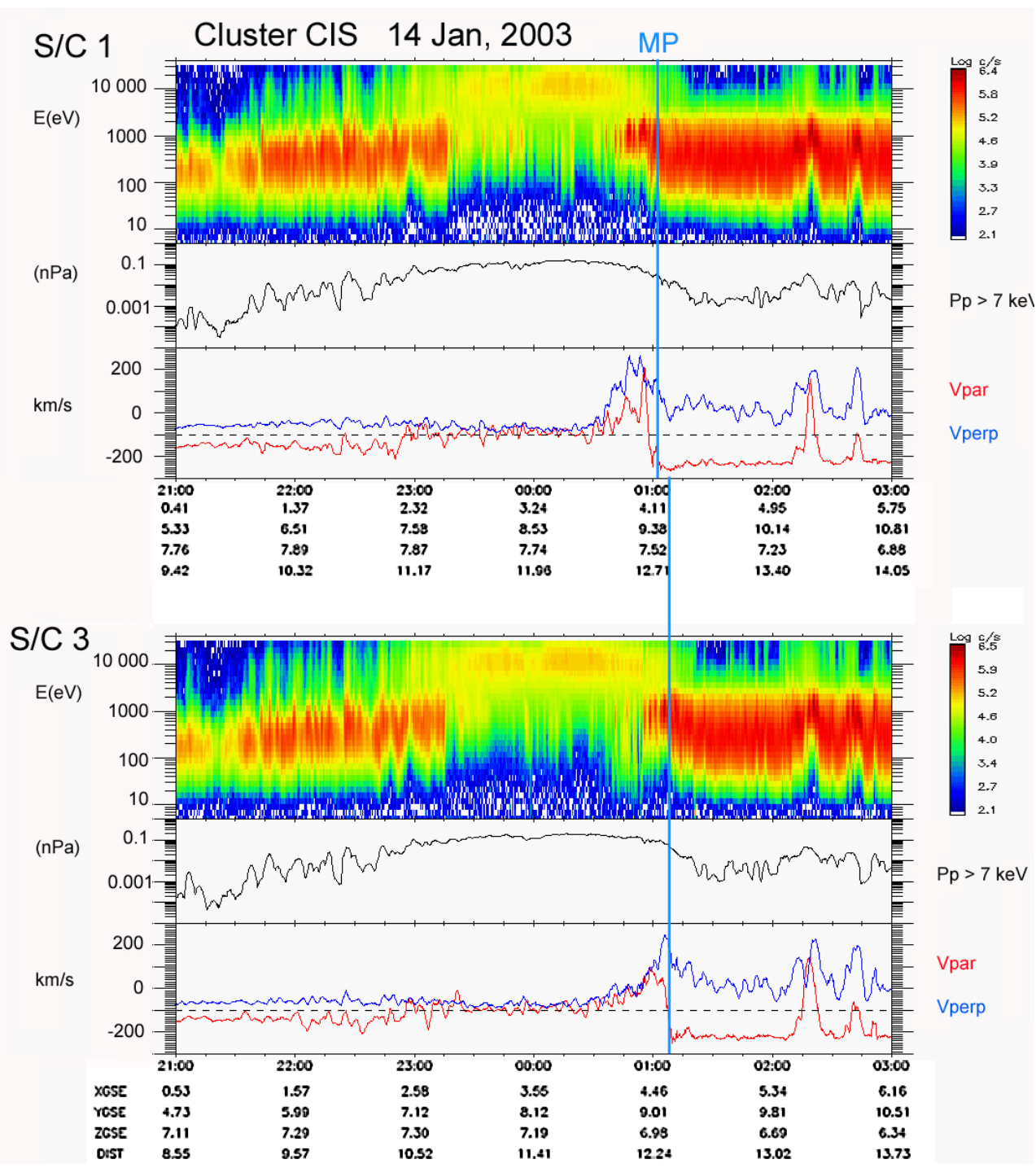

Fig. 2. Overview plot of Cluster s/c 1 and s/c 2 CIS-data on 14 January 2003. The panels illustrate, from top to bottom: energy-time ion spectrograms, ion pressure for energies greater than $10 \mathrm{keV}$, and parallel (red) and perpendicular (blue) ion flow velocities, respectively. The blue line marked "MP1" marks the magnetopause traversal of s/c 1 . Left blue line marks the exit of the cusp.

A further simplification is to take the modulus of $B$. The background magnetic field along the s/c orbit, $\left|B_{S}(t)\right|$, is obtained by fitting the times series of $|B(t)|$ to a power law (trend) function versus time. The rational for choosing a power law function is to minimize the variance of $B$ rather than to mimic a potential model field. From $\left(\left|B_{S}(t)\right|=B_{0}-B_{1}\left(t / t_{0}\right)^{-\alpha}\right.$, where $t$ is time and $B_{0}$ marks the $B$-field at $\left(\mathrm{t}=\mathrm{t}_{0}\right)$ to $\left|B_{S}(t)\right|$, and we obtain:

$|B(t)|=\left|B_{S}(t)\right|+|\Delta B(t)|$,

where $|\Delta B(t)|$ is the modulus of the variance, the local offset $B$. The average magnetic perturbation per unit time is now given by $|\Delta B(t) / \Delta t|$. The $\nabla \times \boldsymbol{E}_{\text {emf }}$ term is obtained by deriving the ion motional emf, $\boldsymbol{E}_{e m f}=-\boldsymbol{v} \times \boldsymbol{B}$, and subsequently taking the $\nabla \times \boldsymbol{E}_{\text {emf }}$ along the s/c orbit. From the modulus of $\nabla \times \boldsymbol{E}_{\text {emf }}$ we obtain $|\Delta B(t) / \Delta t| \approx|\nabla \times(\boldsymbol{v} \times \boldsymbol{B})|$ as our differential frozen-in equation.
Equation (5) implies a high magnetic Reynolds number at a single point. However, that is not sufficient for Eq. (1) to hold because it requires Eq. (5) to hold everywhere within a surface S. Moreover, the surface integration in Eq. (1) must be made over vector fields within the surface $S$, and ideal MHD implies that the sum (integration) of both terms over the surface $\mathrm{S}$ equals zero. The finite differential form for the frozen-in condition requires a normalization factor to scale to the single-point Eq. (5). To understand the geometrical effect we have to examine the integral form, i.e. Eq. (1). Taking now the measured values of $|\Delta B(t) / \Delta t|$ and $|\nabla \times(\boldsymbol{v} \times \boldsymbol{B})|$ and assuming that they remain constant within each surface sample $\Delta S$ along the s/c orbit we derive the following approximation for the change in magnetic flux:

$$
\frac{\Delta \Phi}{d t} \approx\left(\left|\frac{\Delta B}{\Delta t}\right|-\rho|\nabla \times(v \times B)|\right) \cdot \Delta \mathrm{S},
$$



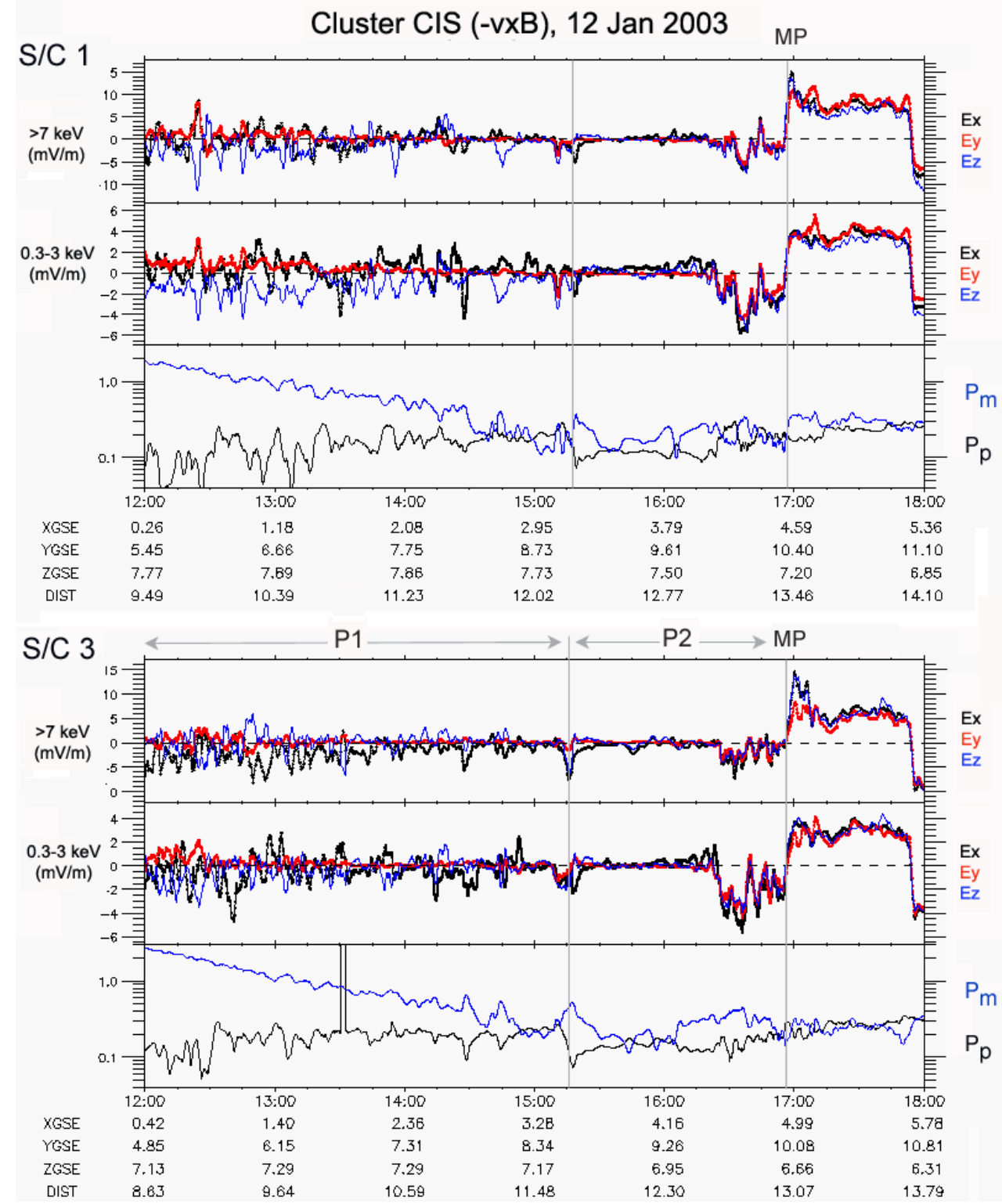

Fig. 3. $-\boldsymbol{v} \times \boldsymbol{B}$ and pressure for s/c 1 and s/c 3 on 12 January 2003. Top panels show $-\boldsymbol{v} \times \boldsymbol{B}$ in GSE coordinates for two energy intervals, $>7 \mathrm{keV}$ and $0.3-3 \mathrm{keV}$. Bottom panel shows plasma pressure $(\mathrm{Pp})$ and magnetic field pressure $(\mathrm{Pm})$.

where $\rho$ is a normalizing constant, which is introduced because $B$ is an integral quantity, while $\nabla \times \boldsymbol{E}_{e m f}$ is spatially distributed, the vector product of $|\nabla \times(\boldsymbol{v} \times \boldsymbol{B})|$ here computed from the in-orbit displacement $(\Delta \mathrm{x}, \Delta \mathrm{y}, \Delta \mathrm{z})$. In effect, this means that the integration over $|\nabla \times(\boldsymbol{v} \times \boldsymbol{B})|$ uses a fixed sampling scale, therefore requiring normalization with $|\Delta B(t) / \Delta t|$. The normalizing constant $\rho$ is determined for each pass under the assumption of ideal MHD in the magnetosheath, that is $\rho \approx|\Delta B(t) / \Delta t| /\left.|\nabla \times|(\boldsymbol{v} \times \boldsymbol{B})\right|_{\text {Sheath }}$. The constant $\rho$ determined in the sheath turns out to give, on average, $\Delta \Phi / d t \approx 0$ in most parts inside the magnetopause, as well. This is quite reasonable considering arguments presented in the next paragraph. However, the local variability of $\Delta \Phi / d t$ may be substantial.
The technique to determine the curl along a s/c orbit was first used for deriving field-aligned currents from magnetic field data (e.g. Ijima and Potemra, 1976). A field-aligned current sheet can be quite accurately determined by using a small correction term, a constant that represents the angle of traversal of the field-aligned current sheet. In a similar manner one may relate $\rho$ with a spatial correction term, with a high $\rho$ implying a slanted traversal of flux tubes/sheets. By introducing a constant $\rho$ (from the cusp into the magnetosheath) we assume a constant angle with which the s/c traverse flux tubes/sheets. This is certainly not inconceivable, considering that the direction of the external (sheet) flow and the internal (boundary layer and cusp) flow is quite similar. Furthermore, the average direction of $\boldsymbol{B}$ remains rather stable within the data time intervals. 


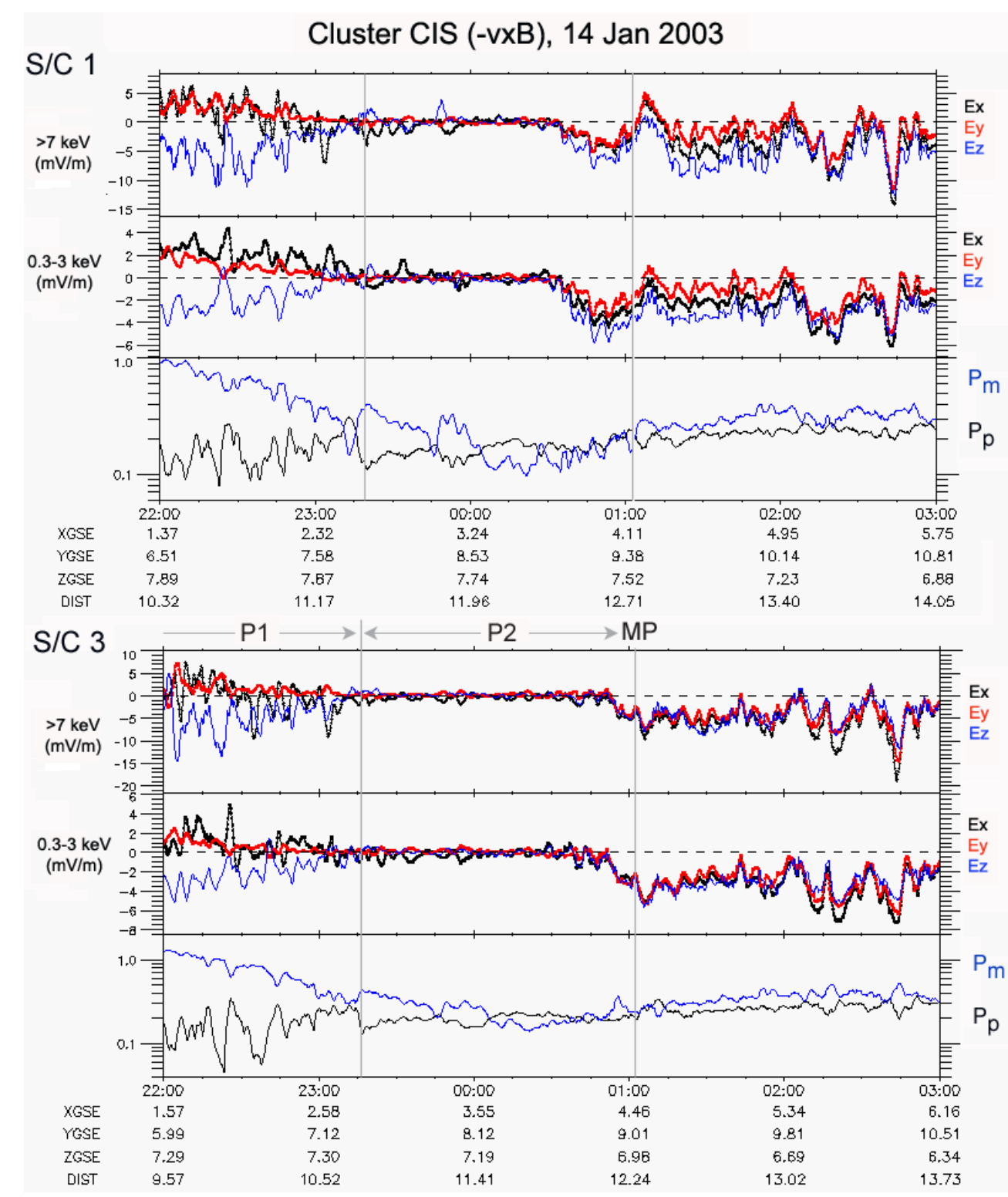

Fig. 4. $-\boldsymbol{v} \times \boldsymbol{B}$ and pressure for s/c 1 and s/c 3 on 14 January 2003. Top panels show $-\boldsymbol{v} \times \boldsymbol{B}$ in GSE coordinates for two energy intervals, $>7 \mathrm{keV}$ and $0.3-3 \mathrm{keV}$. Bottom panel shows plasma pressure $(\mathrm{Pp})$ and magnetic field pressure (Pm).

We have used Cluster CIS and FGM data from 12 January 2003, 10:00-18:00 UT, and 14-15 January, 20:00-04:00 UT for our tests of "ideal MHD" based on Eqs. (3) and (6). In both cases the s/c traversed the outer cusp, entered the magnetospheric boundary layer, and finally went out into the magnetosheath. Figures 1 and 2 show overview plots with energy-time spectrogram (A), ion pressure $>7 \mathrm{keV}$ (B), and parallel and perpendicular velocity (C). Both figures display some general similarities, such as highly variable ion fluxes in the cusp, intermittent injection of magnetosheath ions in the plasma sheet/boundary layer and the intermittent flows of magnetosphere ions in the magnetosheath. The variability of the ion flux and pressure $(>7 \mathrm{keV})$ for both passes may be related to pressure pulses and/or intermittent north-south shifts of the IMF. Notice in Fig. 1 that the magnetopause boundary, encompassed by vertical lines, is crossed almost simultaneously for both s/c, despite a separation distance of $\approx 0.5-1 R_{e}$. Both s/c traverse the early evening sector. From the spacecrafts's GSE X and Y locations one may infer that both $\mathrm{s} / \mathrm{c}$ encountered the magnetopause almost simultaneously, but at different magnetic local times. S/c 3 was located further upstream (of the external sheath flow) compared to $\mathrm{s} / \mathrm{c} 1$. Notice also that the change from cusp to plasma sheet $(\approx 10: 50 \mathrm{UT})$ is also simultaneous for s/c 1 and s/c 3, again an indication that both s/c traversed dayside magnetospheric boundaries almost simultaneously, albeit at different locations. However, it is also evident from Fig. 1 that the small-scale structures embedded in the plasma sheet/boundary layer region $(\approx 11: 00-15: 30$ UT $)$ are quite different for different spacecraft. The latter implies 


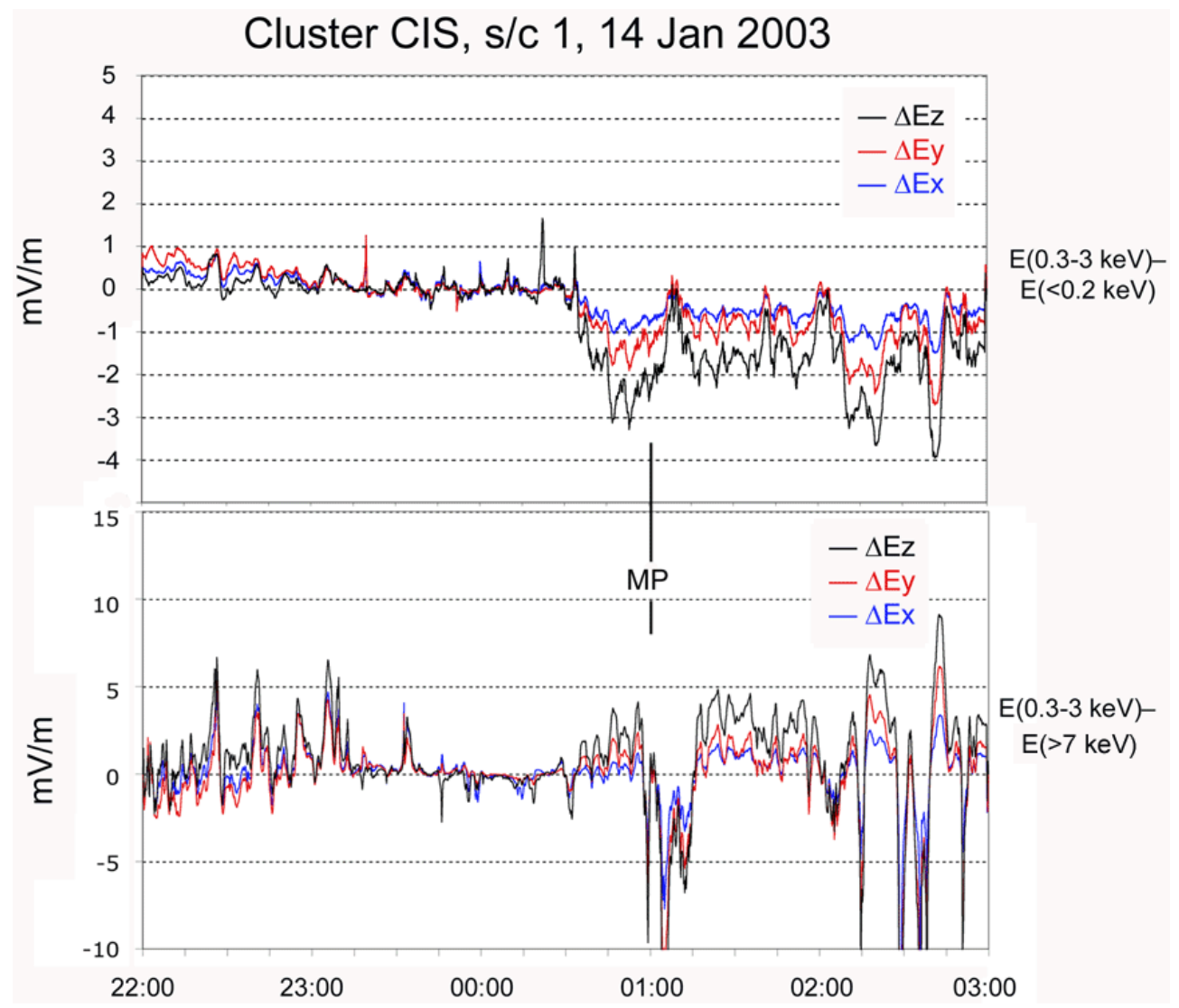

Fig. 5. Cluster s/c 1 ion data on 14 January 2003 of the difference in $-\boldsymbol{v} \times \boldsymbol{B}$ between $0.3-3 \mathrm{keV}$ and $<0.2 \mathrm{keV}\left(\mathrm{E}_{2}-\mathrm{E}_{1}\right)$, and between $0.3-3 \mathrm{keV}$ and $>7 \mathrm{keV}\left(\mathrm{E}_{2}-\mathrm{E}_{3}\right)$, respectively. The magnetopause traversal is marked by MP.

that the embedded structures are clearly temporal. Therefore, we conclude that the magnetospheric boundary regions were rather stably positioned, but the region inside the magnetopause was characterized by transient plasma structures. The plasma transients consisted of a mix of magnetosheath and magnetosphere plasma.

In Figs. 3 and 4 we show ion $-\boldsymbol{v} \times \boldsymbol{B}$ data for two different energy intervals, together with the magnetic and plasma pressure for s/c 1 and s/c 3 during the time period in Figs. 1 and 2. The pressure data illustrates that pressure effects are encountered almost simultaneously for both $\mathrm{s} / \mathrm{c}$. This further validates our assumption that the $\mathrm{s} / \mathrm{c}$ are traversing major boundaries almost simultaneously. The motivation for the differentiation is that ions of different origin, cold ions $(<0.2 \mathrm{keV}$, intermediate energy ions $(0.3-3 \mathrm{keV})$ and energetic ions $(>7 \mathrm{keV})$, may display quite different ion drift properties (Eq. 3), as noted by Lundin et al. (1987, 2003). The variations of $-\boldsymbol{v} \times \boldsymbol{B}$ are in the PC 5 range, suggesting that the $-\boldsymbol{v} \times \boldsymbol{B}$ variations constitute Alfvénic structures. Notice that the PC 5 variability is a persistent feature during both passes, extending into the magnetosheath. It is therefore reasonable to assume that the Alfvénic structures resulted from solar wind pressure pulses or IMF "spikes".

The overall characteristics of the motional emf $-\boldsymbol{v} \times \boldsymbol{B}$ in Figs. 3 and 4 are that of relatively strong $-\boldsymbol{v} \times \boldsymbol{B}$ in the cusp and boundary layer (P1), weaker $-\boldsymbol{v} \times \boldsymbol{B}$ in the plasma sheet (inner P2) and stronger $-\boldsymbol{v} \times \boldsymbol{B}$ in the magnetopause/magnetosheath. A striking feature in the two diagrams is the strong correlation between the $-\boldsymbol{v} \times \boldsymbol{B}$ components in the magnetosheath. In fact, this is one of the most persistent features in the $-\boldsymbol{v} \times \boldsymbol{B}$ data, repeating itself every time the $\mathrm{s} / \mathrm{c}$ enter the magnetosheath. Our assertion is that this is the region that best complies with ideal MHD. Figures 3 and 4 show clear differences in $-\boldsymbol{v} \times \boldsymbol{B}$ for different energy intervals well inside the magnetopause. The differences are indeed significant, as noted in a previous paper (Lundin et al., 2003). Ion flow velocities and the $-\boldsymbol{v} \times \boldsymbol{B}$ terms were determined from the first two moments of the HIA 3-D ion distribution functions. The accumulated counts in the 12-s HIA data separate are quite significant most of the time, even for the limited energy intervals used. The exception is mainly in the tail lobe, where low count rates lead to more erratic flow velocities. Inspecting the colour spectrogram in Figs. 1 and 2 shows that the intermediate and highenergy ranges have adequate count statistics. The moments have been smoothed by a running mean procedure to reduce erratic "noise" in $-\boldsymbol{v} \times \boldsymbol{B}$.

The difference in $-\boldsymbol{v} \times \boldsymbol{B}$ deduced between consecutive energy intervals, i.e. $E_{2}-E_{1}$ and $E_{2}-E_{3}$, where (1) marks $<0.2 \mathrm{keV}$, (2) marks $0.3-3 \mathrm{keV}$, and (3) marks $>7 \mathrm{keV}$, is 


\section{Cluster CIS \& FGM s/c 1; 12 Jan 2003}

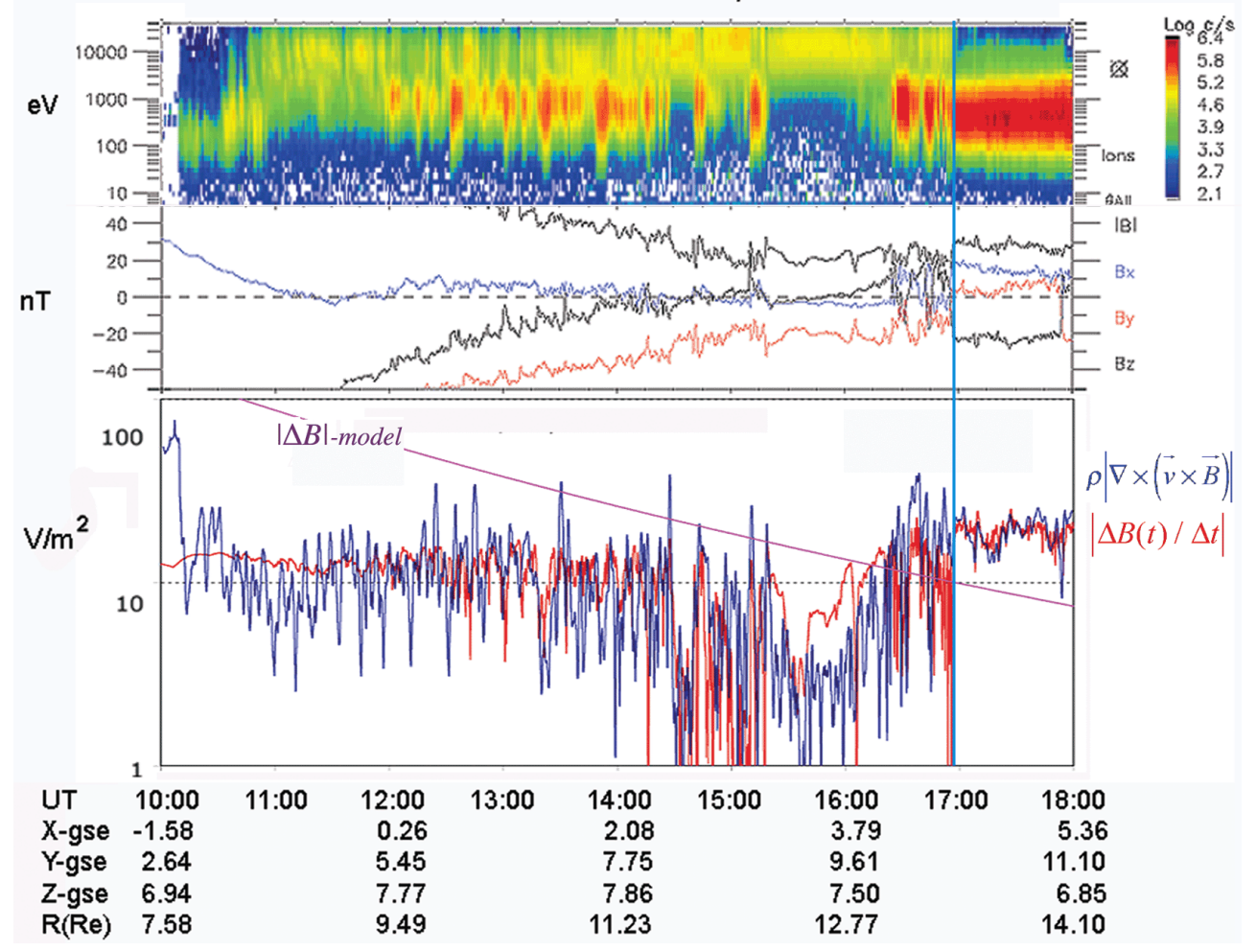

Fig. 6. Cluster s/c 1 CIS and FGM data on 12 January 2003. The panels illustrate, from top to bottom: energy-time ion spectrograms, ion pressure for energies greater than $10 \mathrm{keV}$, and parallel (red) and perpendicular (blue) ion flow velocities, respectively $-\boldsymbol{v} \times \boldsymbol{B}$ from CIS over the entire energy range. Fourth panel gives the magnetic field data. Fifth panel shows $|\Delta \boldsymbol{B} / \Delta t|$ computed by subtracting the model $|B|$ (magenta line) and the $|-\rho| \nabla \times(-\boldsymbol{v} \times \boldsymbol{B}) \mid$ (blue) computed along the orbit $(\rho=3)$. Vertical line marks the magnetopause traversal $(\approx 17: 00 \mathrm{UT})$.

shown in Fig. 5. Notice in the upper panel, $\left(E_{2}-E_{1}\right)$, that the differences are minute in the region adjacent to the magnetopause $(\approx 23: 00-00: 30 \mathrm{UT})$, while the variability/differences is/are much stronger in the lower panel $\left(\mathrm{E}_{2}-\right.$ $\left.\mathrm{E}_{3}\right)$. Moreover, the $\Delta \mathrm{E}$ components in the upper panels are well correlated, suggesting that the modulations are related to MHD-waves. The component differences may be real, but they may also be related to scaling errors because the plasma drift is computed from partial plasma moments. The pronounced $-\boldsymbol{v} \times \boldsymbol{B}$ difference for high energies $(>7 \mathrm{keV})$ is quite different. Here the variability is either uncorrelated or, at best, anti-correlated, indicating that $-\boldsymbol{v} \times \boldsymbol{B}$ for high energy ions is very different from electric drift inside the magnetosphere. However, outside the magnetopause the $\Delta \mathrm{E}$ components are well correlated for all ion energies. Notice that the count rate is low at low energies (Figs. 1 and 2), questions about the significance of $-\boldsymbol{v} \times \boldsymbol{B}$ for $<0.2 \mathrm{keV}$. However, a closer inspection shows that the accumulated counts below $0.2 \mathrm{keV}$ are quite adequate for giving directionality, but less adequate for providing magnitude. The latter is again due to the fact that velocities are computed based on partial moments, with the velocities normalized by partial densities.

The difference in ion drift as observed inside the magnetopause indicates that the flux tubes are unable to maintain their original identities. Convecting flux tubes are expected to evolve, to lose and to gain particles. The breakdown may be local, embedded in an overall pattern of convection. The difference with respect to higher energies $(>7 \mathrm{keV})$ may be partly due to pressure gradients instead of displacement drift, but when drift differences are also observed for different lowenergy intervals it is more likely due to kinetic effects, such as inertia drift. In both cases all the plasma contained in flux tubes cannot be considered "frozen" into the magnetic field. Even if the electrons are "frozen" into the magnetic flux tube, the flux tube has less meaning for ions, so ideal MHD becomes questionable.

The situation is different in the magnetosheath, where the relative differential ion drift is very similar, although the magnitude of the differential $-\boldsymbol{v} \times \boldsymbol{B}$ may be substantial because of the partial density normalization problem. The question is, does this imply that ideal MHD always holds outside the magnetopause? The answer is - not necessarily, because the energetic ion drift is most likely due to pressure gradients and the remaining (dominating) plasma distribution may still behave approximately as an MHD fluid. Therefore, unless one finds a more significant difference in $-\boldsymbol{v} \times \boldsymbol{B}$ for the bulk ion distribution, the ion drift test of ideal MHD is less useful. 


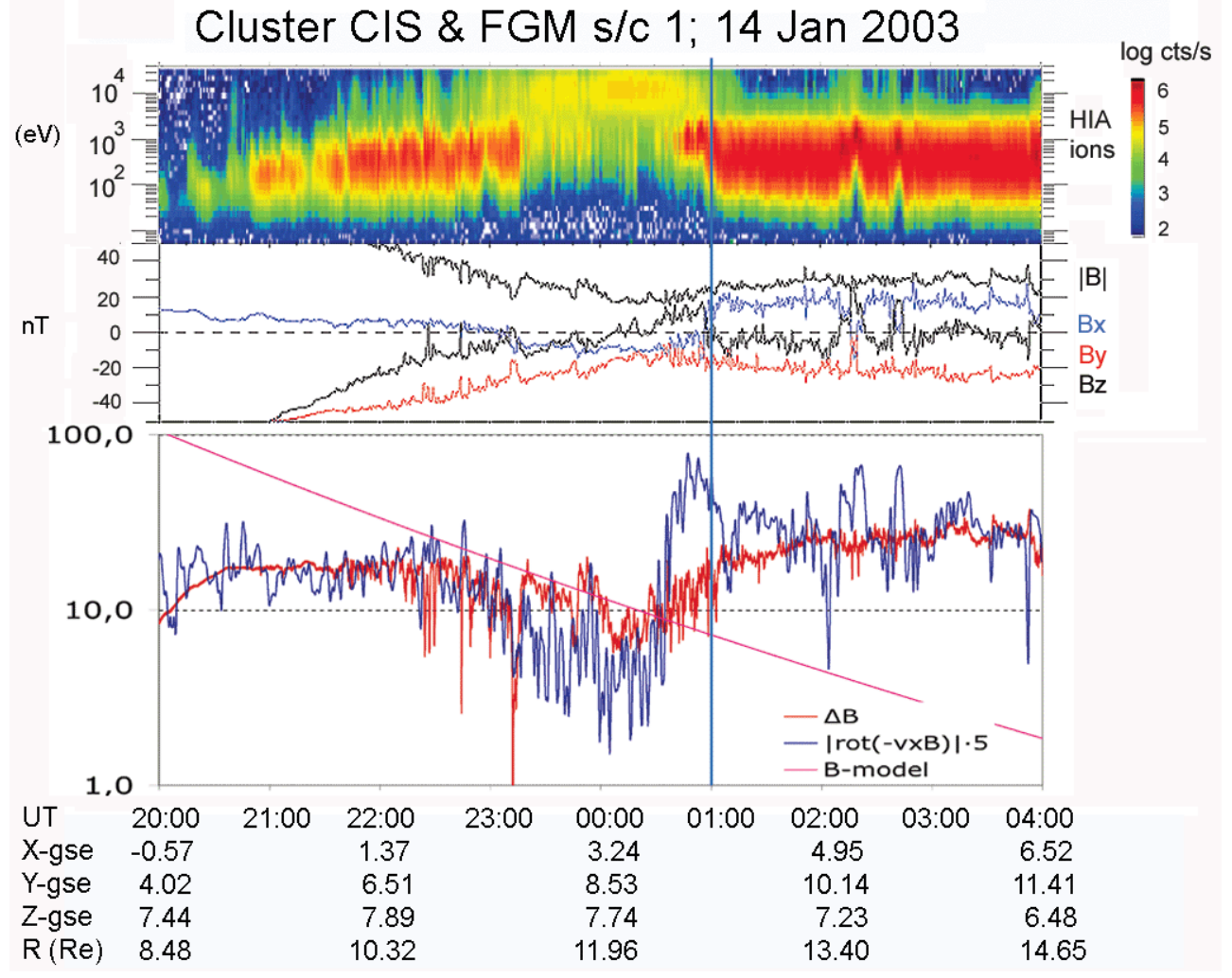

Fig. 7. Cluster s/c 1 CIS and FGM data on 14 January 2003 for "ideal MHD" testing. The format is the same as in Fig. 6 ( $\rho=5$ ). Vertical line marks the magnetopause traversal $(\approx 01: 00 \mathrm{UT})$.

A test based on the "frozen-in" theorem, as described above, may serve the purposes better. Given the data from the two cases analyzed in more detail, we compute the $\nabla \times \boldsymbol{E}_{\text {emf }}$ along the spacecraft orbit. To test if ideal MHD applies for the plasma ensemble, we determine $-\boldsymbol{v} \times \boldsymbol{B}$ from CIS over the entire energy range. We also determine the $\partial B / \partial t$ term by subtracting the "baseline" of $B$ originating from the Earth's dipole plus other quasi-steady magnetic fields induced by currents (e.g. the magnetopause current). On the basis of Eq. (5) we may now plot the two test parameters $|\Delta B(t) / \Delta t|$ and $\rho|\nabla \times(\boldsymbol{v} \times \boldsymbol{B})|$ versus time, as shown in Figs. 6 and 7. Notice that the normalizing constant, $\rho$, is chosen to minimize the variance in the magnetosheath where ideal MHD is assumed to hold, i.e. the change in magnetic flux approaches zero. Figure 6 demonstrates a general agreement between $|\Delta B(t) / \Delta t|$ and $\rho|\nabla \times(\boldsymbol{v} \times \boldsymbol{B})|$ along the orbit, with the main deviations observed in small-scale structures for a limited time period between $\approx 15: 00-16: 00 \mathrm{UT}$, where $|\Delta B(t) / \Delta t|$ is higher. Similarly, we find an overall agreement between values in Fig. 7, this time with $\rho|\nabla \times(\boldsymbol{v} \times \boldsymbol{B})|$ higher at $\approx 00: 30-02: 00$ UT.

The overall agreement along the orbit, with the exception of the innermost (lobe) region in Fig. 7 and the periods mentioned above, suggests that ideal MHD holds in a large-scale sense within the magnetosphere. Departure from ideal MHD is most obvious in plasma transients and in the region immediately inside the magnetopause. Figure 8 shows the departure in more detail, with the variability between $|\Delta B(t) / \Delta t|$ and $\rho|\nabla \times(\boldsymbol{v} \times \boldsymbol{B})|$ sometimes displaying complex phase shifts. There may be at least two reasons for the fact that $\Delta \Phi / d t$ is nonzero within transients. The first is that ideal MHD is indeed invalid. The second is that "wavetrains" of plasma are traversed, such that the assumption of a constant $\rho$ cannot be used. However, in the latter case, it implies that $\rho$ must vary quasi-periodically between $\approx 5$ and 20 . On the other hand, the extended period during the magnetopause approach in Fig. $8(\approx 00: 30-01: 00$ UT) is also characterized by nonzero $\Delta \Phi / d t$, but now in an opposite sense with regard to $\rho$. It is therefore very difficult to conceive a "baseline" $\rho$ that leads to $d \Phi / d t \approx 0$.

\section{Discussions and conclusions}

We have presented two tests of the frozen-in magnetic field theorem. One test is based on the existence of differential ion drift, i.e. the difference in perpendicular drift for ions of different origin. Whenever ions of different origin are contained in a magnetic flux tube, the ions drifting in different directions and with different perpendicular drift speed, the flux tube concept is not valid for the entire plasma ensemble. In effect, this means that the ion drift is not entirely due to the convection electric field; other terms as described by Eq. (3) have to be involved. This has been known for 


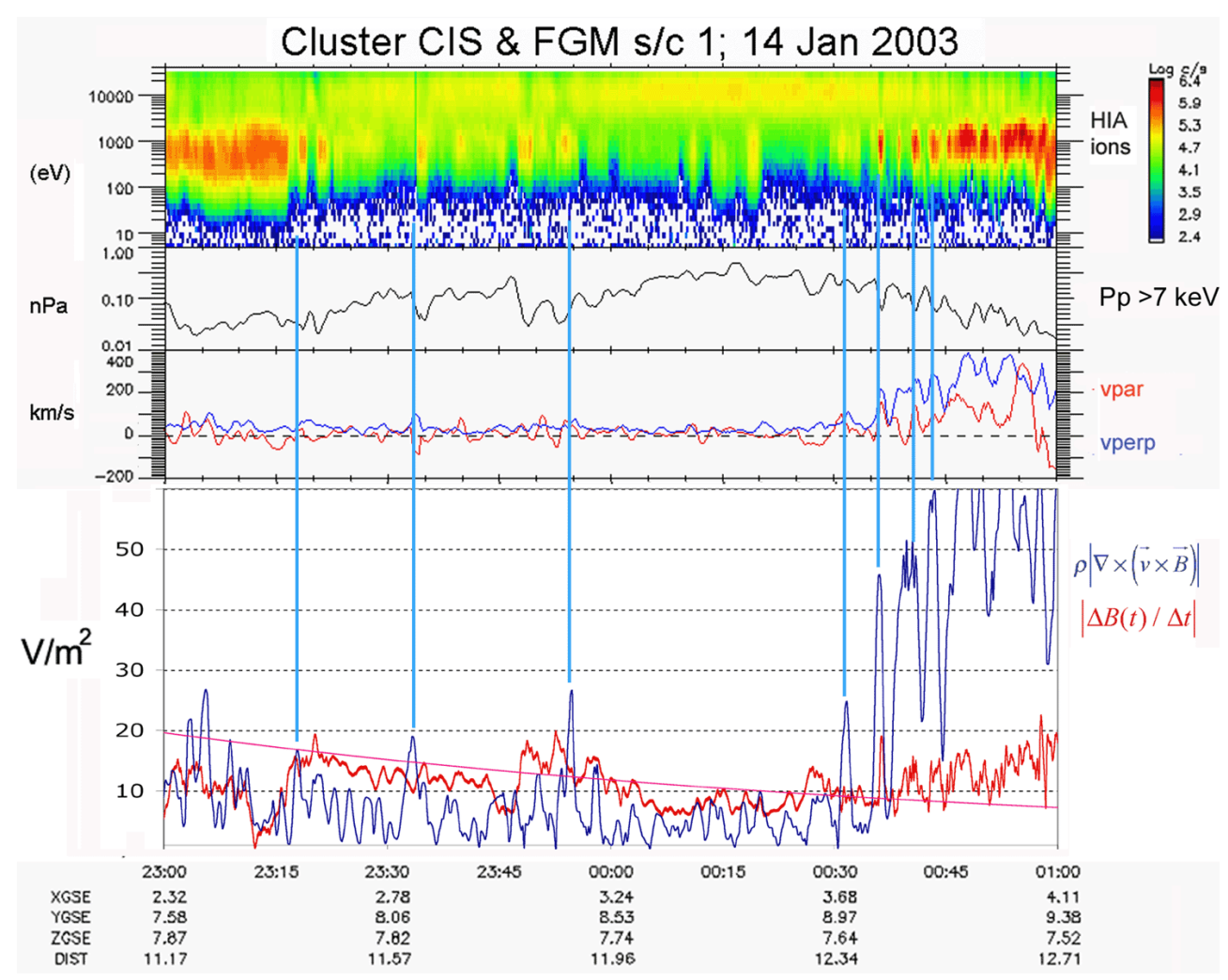

Fig. 8. Detailed view of the ideal MHD-test on 14 January 2004, showing the departure of ideal MHD in periods of plasma transients and on a meso/large scale at the magnetopause approach. Vertical lines mark peaks in the $|-\rho| \nabla \times(-\boldsymbol{v} \times \boldsymbol{B}) \mid$ component and their interrelation with plasma transients. Notice the dramatic rise of $|-\rho| \nabla \times(-\boldsymbol{v} \times \boldsymbol{B}) \mid$ in the magnetopause layer.

a long time in magnetospheric physics, as illustrated by the difference in drift patterns for high-energy and low-energy ions subject to combined gradient curvature drift and electric drift. Hot multi-component plasmas in the magnetosphere boundary layer display similar properties (e.g. Lundin et al., 1987, 2003). Even a dense magnetized plasma of electrons and cold ions may depart from ideal MHD, if a sufficient admixture of hot ions is introduced. The question is, to what extent does an admixture of different plasma components with different drift signatures affect ideal MHD? The answer to this question must be obtained from experimental tests of the requirement for ideal MHD - proving/disproving that $\Delta \Phi / d t$ is close to zero within a flux tube.

Experimentally testing the frozen-in field theorem requires magnetic field and plasma measurements within a two-dimensional cut of a magnetic flux tube, a task within the framework of the Cluster mission objectives. However, to produce adequate data for fields and particles turned out to be quite difficult using only four $\mathrm{s} / \mathrm{c}$. In this report we have used plasma data from three spacecraft to determine the overall/large-scale morphology of the boundary regions transited, but we only use one $\mathrm{s} / \mathrm{c}$ at a time to determine $\Delta \Phi / d t$, as described above. On the basis of these results we argue that ideal MHD applies in an average sense on larger scales, i.e. $\Delta \Phi / d t \approx 0$ if averaged over larger spatial volumes in the magnetosphere. Moreover, perhaps not surprising, the quasi-steady magnetosheath complies well with ideal MHD, also on fine scales.

Near the magnetopause we find broad regions of departure from ideal MHD, with $\Delta \Phi / d t \neq 0$. We note that a nonzero $\Delta \Phi / d t$ implies electromotive forcing, i.e. a transfer of energy from fields to particles, or vice versa. $\Delta \Phi / d t>0$ implies that magnetic induction dominates at the expense of the kinetic/electric forcing, according to the Faraday-Henry's law. Similarly, when $\Delta \Phi / d t<0$, kinetic/electric forcing dominates at the expense of magnetic induction, i.e. energy goes from particles to fields. From Figs. 6 and 7 we note that the region immediately inside the magnetopause is characterized by $\Delta \Phi / d t<0$, implying that particle kinetic energy goes into electromagnetic energy (i.e. deceleration and/or release of pressure gradient). Further inside the magnetopause we find the opposite, $\Delta \Phi / d t>0$. This may indicate that magnetic forcing dominates and an emf $\left(E_{e m f}\right)$ is induced by the changing magnetic flux. However, the significance of $\Delta \Phi / d t>0$ may be questioned here because just a minor increase in $\rho$ will make $\Delta \Phi / d t \approx 0$; of more importance is rather that it further validates that an extended region of $d \Phi / d t<0$ is found immediately inside the magnetopause.

Inspecting the time series plot of $|\nabla \times(\boldsymbol{v} \times \boldsymbol{B})|$ and $\rho|\nabla \times(\boldsymbol{v} \times \boldsymbol{B})|$ in greater detail (Fig. 8), we find that the plasma transients well inside the magnetopause are characterized by modulating differences/departures up to an 


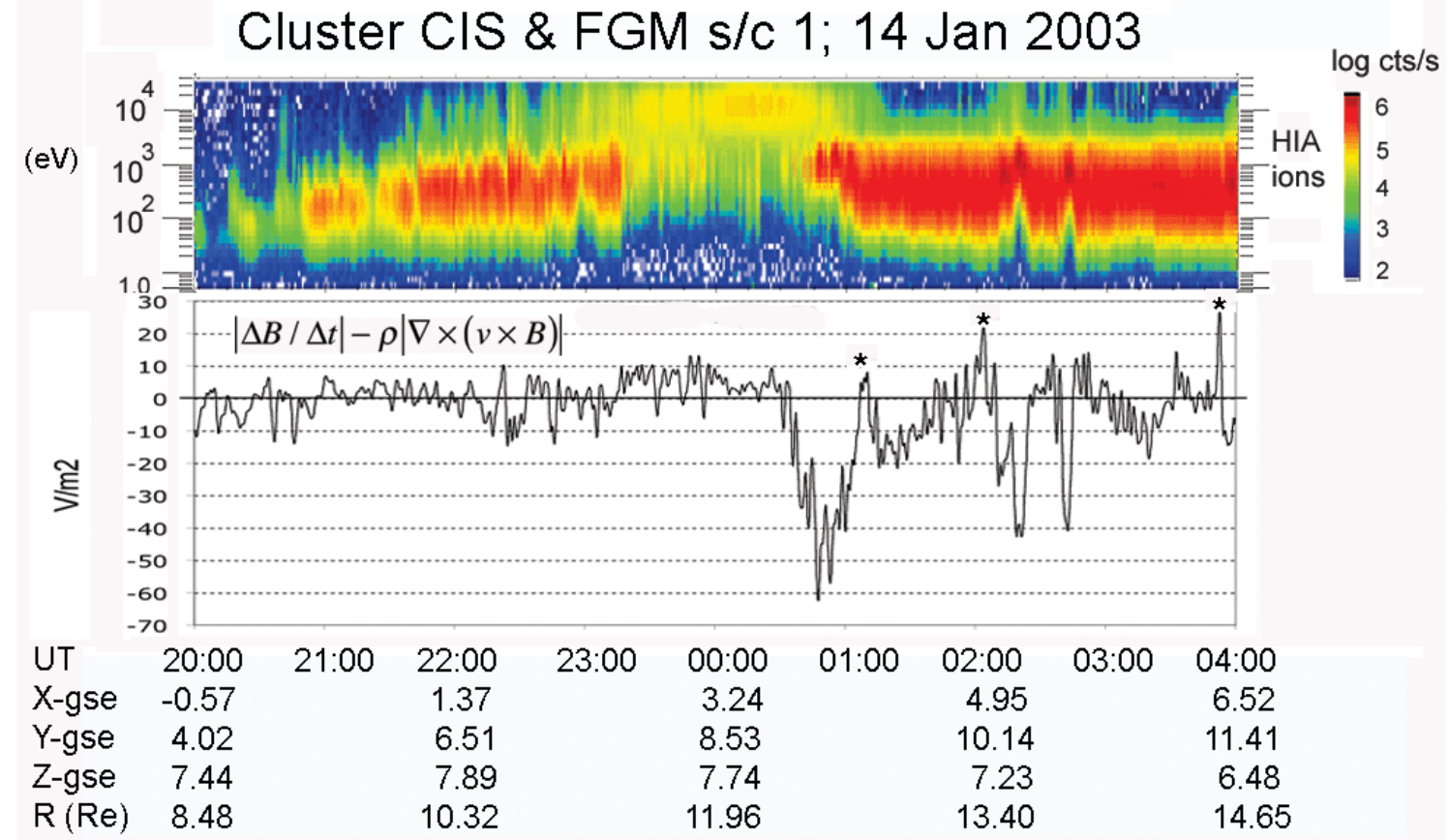

Fig. 9. Graph illustrating $|\Delta B / \Delta t|-\rho|\nabla \times(-\boldsymbol{v} \times \boldsymbol{B})|$ (Eq. 6) versus time for the 14 January 2003 Cluster traversal. Negative values near the magnetopause $(\approx 00: 30-01: 00 \mathrm{UT})$, i.e. $d \Phi / d t<0$ (diamagnetic effects), imply significant departure from ideal MHD and strong plasma forcing. $d \Phi / d t>0$ (magnetic induction) occurs during $\approx 23: 00-00: 30$ UT. The magnetopause is traversed at $\approx 01: 00 \mathrm{UT}$.

order of magnitude. The modulations of $|\nabla \times(\boldsymbol{v} \times \boldsymbol{B})|$ and $\rho|\nabla \times(\boldsymbol{v} \times \boldsymbol{B})|$ are quite complex, in phase or in antiphase. Figure 8 displays a stable low-frequency modulation in the $\mathrm{PC} 1$ (tens of $\mathrm{mHz}$ ) range superimposed on a low-frequency modulation in the PC5 range. The amplitude of both modulations are similar up to the magnetopause layer (23:0000:30 UT $)$, where the $\rho|\nabla \times(\boldsymbol{v} \times \boldsymbol{B})|$-term dominates.

Further inspection of Fig. 8 reveals that each plasma transient is associated with an enhanced $\rho|\nabla \times(\boldsymbol{v} \times \boldsymbol{B})|$. This is again confirming that the transient carries excess energy and momentum. The simultaneous enhancement of $|\nabla \times(\boldsymbol{v} \times \boldsymbol{B})|$ suggests magnetic perturbations generated by electric currents and/or time dependent electric fields. This is in agreement with the picture of localized plasma entry into the magnetosphere, and the associated energy and momentum transfer generating fields and electric currents. Whether the entry itself is governed by transient or steady-state reconnection (e.g. Paschmann et al., 1979; Russell and Elphic, 1979; Russel, 1981; Sonnerup et al., 1981) or by other processes governing entry through the magnetopause (e.g. Lemaire, 1977; Lemaire and Roth, 1978; Echim and Lemaire, 2000) cannot be determined from this test.

Figures 9 and 10 finally show the normalized change in magnetic flux for the Cluster passes, using Eq. (6), i.e. $\Delta \Phi /(\Delta t \cdot \Delta S) \approx(|\Delta B / \Delta t|-\rho|\nabla \times(v \times B)|)$. Notice from Fig. 9 that the time period $\approx 23: 15-00: 30$ UT is characterized by a relatively constant and positive $\Delta \Phi /(\Delta t \cdot \Delta S)$, while the period 00:30-01:00 UT is persistently negative, up to $-60 \mathrm{~V} / \mathrm{m}^{2}$. The latter is consistent with strong plasma forcing, effectively a source of energy and momentum. The excess energy may go into wave turbulence, as observed near the magnetopause by André et al. (2001) and Vaivads et al. (2004), or to electric currents feeding energy into the dayside ionosphere. A third option is that particle kinetic energy is locally redistributed (e.g. converted to field-aligned plasma flow). Figure 10 displays similar features as Fig. 9, i.e. a slightly positive $\Delta \Phi /(\Delta t \cdot \Delta S)$ between $\approx 14: 00-16: 00$ UT when the s/c encountered plasma sheet ions, and negative within transients and near the magnetopause $(\approx 16: 30$ 17:00 UT). A common feature in both Cluster cases presented here is that the magnetopause region is characterized by $d \Phi / d t<0$, which implies that plasma/particle forcing dominates over electromagnetic forcing. Plasma transients inside the magnetopause behave in a more complicated way, with strong variations between positive and negative $d \Phi / d t$. On the other hand, the trend is $d \Phi / d t>0$ for the region well inside the magnetopause with plasma sheet ions, suggesting electromagnetic forcing of the plasma. Narrow regions found near and outside of the magnetopause with strong $d \Phi / d t>0$ (marked by * in Figs. 9 and 10) are believed to be associated with electromagnetic forcing.

The drawback of an analysis like this, which uses single spacecraft measurements, is, of course, the ambiguity between temporal and spatial. The cases were selected to minimize this ambiguity. For instance, the magnetopause and other pronounced features (pressure gradients) were crossed almost simultaneously, albeit at different local times, by the three Cluster s/c (1, 3 and 4). This shows that transient features in the boundary layer were really localized and time dependent, while the magnetopause remained quite stable. 


\section{Cluster CIS \& FGM s/c 1; 12 Jan 2003}
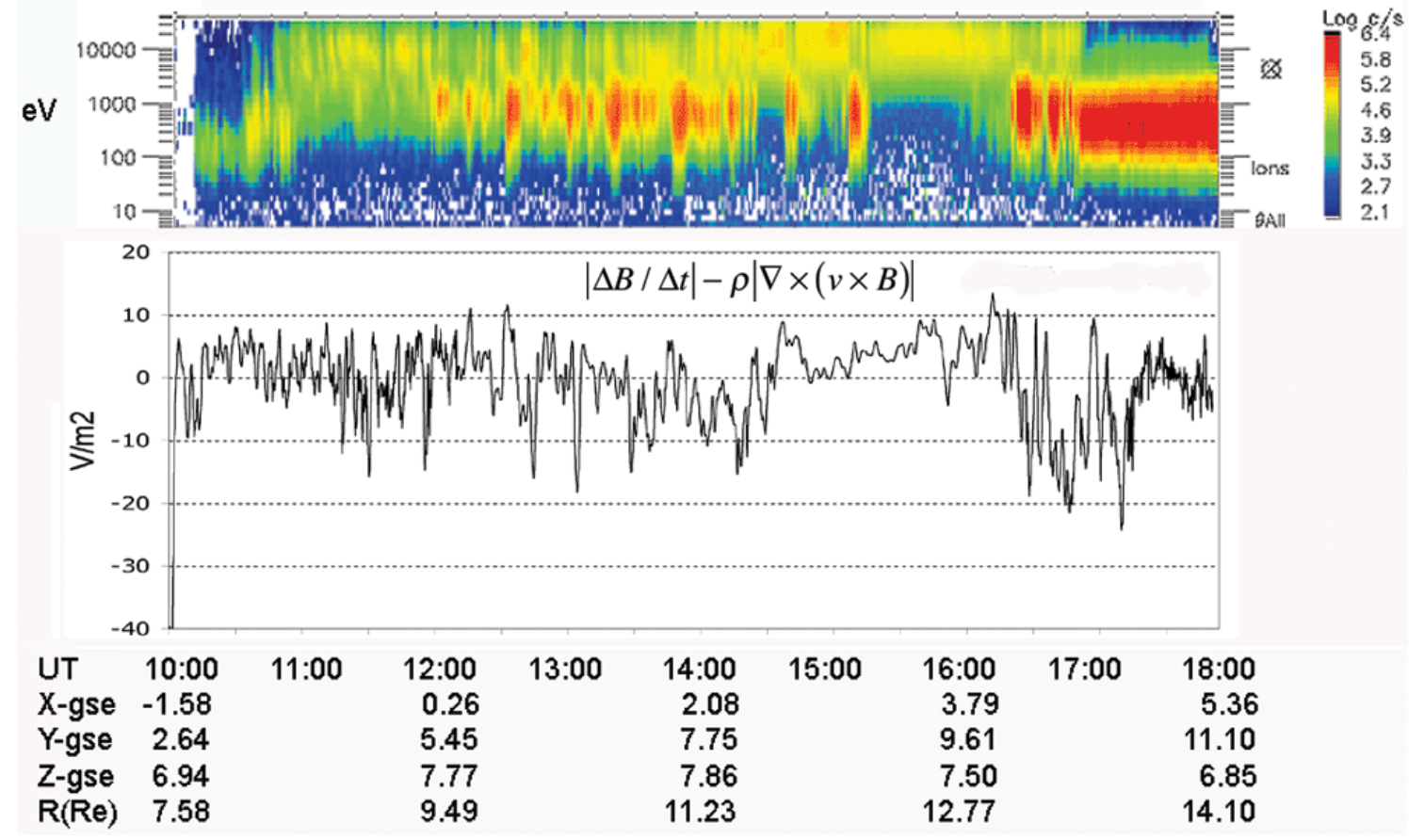

Fig. 10. Graph illustrating $|\Delta \boldsymbol{B} / \Delta t|-\rho|\nabla \times(-\boldsymbol{v} \times \boldsymbol{B})|$ (Eq. 6) versus time for the 12 January 2003 Cluster case. The magnetopause is traversed at $\approx 17: 00 \mathrm{UT}$.

We therefore interpret the temporal-spatial ambiguity illustrated by $d / d t=\delta / \delta t+(\boldsymbol{u} \cdot \nabla)$, such that the large-scale variability of $B$ is dominated by the $\delta B / d t$ term, while boundary layer transients are also affected by the $(\boldsymbol{u} \boldsymbol{\nabla})$ term. The latter implies a smaller $\delta B / d t$-term in transients. This suggests lower mean values of $|\Delta B(t) / \Delta t|$ during the time interval 23:15-00:30 UT in Fig. 8, values closer to the mean value of $\rho|\nabla \times(\boldsymbol{v} \times \boldsymbol{B})|$. Amplitude differences remains unaffected, though. The opposite holds for the time interval 00:3001:00 UT (Fig. 8). Only temporal variations of $-\boldsymbol{v} \times \boldsymbol{B}$ without coincident temporal variations of $\delta B / d t$ can possibly explain that $\rho|\nabla \times(\boldsymbol{v} \times \boldsymbol{B})|$ is so much larger (up to ten times) than $|\Delta B(t) / \Delta t|$. Magnetic perturbations, whether spatial or temporal, are simply too small to balance the $\rho|\nabla \times(\boldsymbol{v} \times \boldsymbol{B})|$ term.

Based on the MHD test described above we make the following conclusions:

- Ideal MHD, i.e. $d \Phi / d t \approx 0$, appears to apply on large scales in most parts of the exterior magnetosphere and in the "undisturbed" magnetosheath.

- Departure from ideal MHD, nonzero $d \Phi / d t$, implies electromotive forcing (Faraday-Henry's law), i.e. excess energy and momentum may be transferred from particles to fields, or vice versa.

- Departure from ideal MHD in extended regions (several thousand $\mathrm{km}$ ) is observed near the magnetopause. Our two examples suggests that $d \Phi / d t$ is negative at, or in the immediate vicinity of, the magnetopause. A negative $d \Phi / d t$ implies induction, i.e. $\nabla \times(\boldsymbol{v} \times \boldsymbol{B})>d \boldsymbol{B} / d t$. Such forcing is capable of producing (electro)magnetic energy at the expense of plasma kinetic energy.

- Further inside the magnetopause we also identify regions where $d \Phi / d t$ is persistently positive. A positive $d \Phi / d t$ implies electromagnetic forcing, i.e. energy may go from fields to particles.

- Departure from ideal MHD is also observed on smaller scales in magnetosheath plasma transients inside the magnetopause. Plasma transients bear the magnetic and $-\boldsymbol{v} \times \boldsymbol{B}$ signatures of FTEs (Russell and Elphic, 1979) and/or PTEs (Lundin, 1988; Woch and Lundin, 1992). $d \Phi / d t$ in plasma transients varies quasi-periodically with periodicities in the Pc 1 to Pc 5 range, suggesting that they constitute Alfvénic structures.

Ideal MHD $(d \Phi / d t \approx 0)$ is certainly useful for transport applications where convection plays the dominant role for magnetized plasmas, like the cold plasma in the Earth's magnetosphere and ionosphere. However, departure from "ideal MHD”, as observed in the terrestrial hot magnetospheric plasma, is a memento for simple convection models. Departure from ideal MHD implies electromotive forcing driven by, for examle, plasma inertia-effects, plasma-gradients and field anisotropies, effects that have to be adequately considered in space plasmas. Departure from ideal MHD also means that plasma may access through the magnetopause, 
meaning in reconnection terminology, accessing via a diffusion region.

Understanding non-ideal MHD is therefore critical for understanding energy and momentum transfer in space plasmas. But also transport in space plasmas is an issue with bearing on ideal MHD, for instance, what part is due to convection and what part is due to other drift processes? The tool presented here may help elucidate these issues. To further analyze the spatial temporal ambiguity, this tool may be refined and tested on data from periods when the Cluster $\mathrm{s} / \mathrm{c}$ were in a closer formation. However, the Cluster data set is still expected to be insufficient to finally prove or disprove this intriguing and most relevant issue in contemporary space plasma physics. A new and more adequate multi-spacecraft mission providing three-dimensional measurements at different characteristic scale-sizes, is required to resolve this critical issue.

Acknowledgements. The authors are indebted to C.-G. Fälthammar for comments and constructive criticism of the paper. The Cluster project was managed and funded by the European Space Agency. The French part of Cluster CIS was funded by CNES and CNRS, while the Swedish contribution was funded in part by the Swedish National Space Board and the Wallenberg Foundation.

Topical Editor T. Pulkkinen thanks K. Shikawa and another referee for their help in evaluating this paper.

\section{References}

Alfvén, H.: A theory of the magnetic storms and the aurorae II and III, Kgl. Sv. Vet. ak. Handl. Tredje Ser., Band 18, no 9, 1940.

Alfvén, H.: Existence of electromagnetic-hydrodynamic waves, Nature, vol. 150, 405, 1942.

Alfvén, H.: Cosmical Electrodynamics, Oxford University Press, 1950.

Alfvén, H.: On the theory of magnetic storms and aurorae, Tellus, 10, 104-116, 1958.

Alfvén, H.: On frozen-in field lines and field-line reconnection, J. Geophys Res, 81, 4019-4021, 1976.

Alfvén, H.: Cosmic plasma, Astrophys and Space Science Library, Vol. 82, D. Reidel, Dordrecht, Holland, 1981.

Alfvén, H.: Paradigm Transition in Cosmic Plasma Physics, Geophys. Res. Lett., 10, 487-488, 1983.

Alfvén, H. and Fälthammar, C.-G.: Cosmical Electrodynamics, Int. Series of Monographs on Physics, Clarendon Press, Oxford, 1963.

André, M., Behlke, R., Wahlund, J.-E., Vaivads, A., Eriksson, A.-I., Tjulin, A., Carozzi, T. D., Cully, C., Gustafsson, G., Sundkvist, D., Khotyaintsev, Y., Cornilleau-Wehrlin, N., Rezeau, L., Maksimovic, M., Lucek, E., Balogh, A., Dunlop, M., Lindqvist, P.-A., Mozer, F., Pedersen, A., Fazakerley, A.: Multi-spacecraft observations of broadband waves near the lower hybrid frequency at the Earthward edge of the magnetopause, Ann. Geophys., 19, 1471-1481, 2001,

SRef-ID: 1432-0576/ag/2001-19-1471.

Cole, K. D.: On solar wind generation of polar magnetic disturbances, J. Astron. Soc., 4, 103, 1961.

Chen, F. F. (Ed.): Introduction to plasma physics and controlled fusion, Volume 1, Plenum Press, 1984.
Dungey, J. W.: Interplanetary fields and the auroral zone, Phys. Rev. Lett., 6, 47-48, 1961.

Echim, M. M. and Lemaire, J. F.: Laboratory and numerical simulations of the impulsive penetration mechanism, Space Sci. Rev., 92, 565-601, 2000.

Hall, D. S., Hapgood, M. A., and Bryant, D. A.: The transition from the magnetosheath to the magnetosphere, Geophys. Res. Lett., 17, 2039-2042, 1990.

Iijima, T. and Potemra, T. A.: Field aligned currents in the dayside cusp observed by Triad, J. Geophys. Res., 81, 5971-5979 1976.

Lemaire, J.: Impulsive penetration of filamentary plasma elements into the magnetospheres of the Earth and Jupiter, Planet Space Sci., 25, 887-890, 1977.

Lemaire J. and Roth, M.: Penetration of solar wind plasma elements into the magnetosphere, J. Atmos. Terr. Phys., 40, 331335, 1978.

Lundin, R.: On the magnetospheric boundary layer and solar wind energy transfer into the magnetosphere, Space Sci. Rev., 48 , 263-320, 1988.

Lundin, R., Stasiewicz, K., and Hultqvist B.: On the interpretation of different flow vectors of different ion species in the magnetospheric boundary layer, J. Geophys. Res., 92, 3214-3222, 1987.

Lundin, R., Sauvaud, J.-A., Rème, H., Balogh, A., et al.: Evidence for impulsive solar wind plasma penetration through the dayside magnetopause, Ann. Geophys., 21, 457-472, 2003,

SRef-ID: 1432-0576/ag/2003-21-457.

Mozer, F. S., Bale, S. D., and Phan, T. D.: Evidence of Diffusion Regions at a Subsolar Magnetopause Crossing, Phys. Rev. Lett., 89, 015002, 2002.

Northrop, T. G.: The adiabatic motion of charged particles, John Wiley, New York, 1963.

Parker, E. N.: Newtonian development of the dynamical properties of ionized gases of low density, Phys. Rev., 107, 924-933, 1957a.

Parker, E. N.: Sweet's mechanism for merging magnetic fields in conducting fluids, J. Geophys. Res., 62, 509-520, 1957 b.

Phan, T.-D. and Paschman, G.: Low-latitude dayside magnetopause and boundary layer for high magnetic shear, J. Geophys. Res., 101, 7801-7816, 1996.

Paschmann, G., Ö.Sonnerup, B. U., Papamastorakis, I., Sckopke, N., Haerendel, G., Bame, S. J., Asbridge, J. R., Gosling, J. T., Russell, C. T., and Elphic, R. C.: Plasma acceleration at the earth's magnetopause: Evidence for reconnection, Nature Lond., 282, 243-246, 1979.

Paschmann, G. and Treumann, R.: Auroral Plasma Physic, edited by: Paschmann, G., Haaland, S., Treumann, R., ISSI Space Science Series, Vol 15, ISBN 1-4020-0963-1, 2003.

Rème, H., Aoustin, C., Bosqued, J. M., Dandouras, I. et al.: First multispacecraft ion measurements in and near the Earth's magnetosphere with the identical Cluster ion spectrometry (CIS) experiment, Ann. Geophys., 19, 1303-1354, 2001,

SRef-ID: 1432-0576/ag/2001-19-1303.

Russell, C. T.: The magnetopause of the earth and planets, Advances in Space Research, 1, 1, 67-87, 1981.

Russell, C. T. and Elphic, R. C.: ISEE observations of flux transfer events at the dayside magnetopause, Geophys. Res. Lett., 6, 3336, 1979.

Russell, C. T.: Reconnection at the Earth's Magnetopause: Magnetic Field Observations and Flux Transfer Events, Magnetic Reconnection in Space and Laboratory Plasmas: Geophysical Monograph 30, edited by: Hones Jr., E. W., American Geophysical Union, Washington D.C., 124, 1984. 
Schindler, K., Hesse, M., and Birn, J.: General magnetic reconnection, parallel electric fields, and helicity, J. Geophys. Res., 93, 5547-5557, 1988.

Sonnerup, B. U. Ö.: Theory of the low-latitude boundary layer, J. Geophys. Res., 85, 2017-2026 1980.

Sonnerup, B. U. Ö., Paschmann, G., Papamastorakis, I., Sckopke, N., Haerendel, G., Bame, S. J., Asbridge, J. R., Gosling, J. T., and Russell, C. T.: Evidence for magnetic field reconnection at the Earth's magnetopause, J. Geophys. Res.,86, 10 049-10067, 1981.

Stasiewicz, K.: A global model of gyroviscous field line merging at the magnetopause, J. Geophys. Res., 96, 77-86, 1991.

Sweet, P. A.:, The neutral point theory of solar flares, in: Electromagetic Phenomena in Cosmical Physics, edited by: Lehnert, B., Cambridge University Press, London, 1958.

Vaivads, A., André, M., Buchert, S., Wahlund, J.-E., Fazakerley, A., and Cornilleau-Wehrlin, N.: Cluster Observations of lower hybrid turbulence at the magnetopause, Geophys. Res. Lett., 31, doi:10.1029/2003GL018142, 2004.
Vasyliunas, V. M.: Theoretical models of magnetic field line merging, 1, Rev. Geophys., 13, 303-336, 1975.

Woch, J. and Lundin, R.: Signatures of transient boundary layer processes observed with Viking, J. Geophys. Res., 1431-1447, 1992.

Yamauchi, M. and Blomberg, L.: Problems on mappings of the convection and on the fluid concept, Phys. Chem. Earth, 22, 709714, 1997.

Yamauchi, M., Lundin, R., Norberg, O., Sandahl, I., Eliasson, L., and Winningham, D.: Signature of direct magnetosheath plasma injections onto closed field-line regions based on observations at mid- and low-altitudes, in: Earth's Low-Latitude Boundary Layer, edited by: Newell, P. T. and Onsager, T., AGU monograph, 179-188, 2003. 